\title{
Effect of spinal irradiation on growth
}

\author{
S M SHALET, B GIBSON, R SWINDELL, AND D PEARSON \\ Departments of Endocrinology, Radiotherapy, and Statistics, Christie Hospital and Holt Radium Institute, \\ Manchester, and Department of Paediatric Haematology, Royal Hospital for Sick Children, Glasgow
}

SUMMARY Standing height, sitting height, and leg length were measured in 79 patients (aged $16-30$ years), who had been given craniospinal irradiation $(n=37)$ or cranial irradiation $(n=42)$ in childhood for a brain tumour and had completed their growth. Their measurements were compared with established standards for sitting height and leg length in British children (aged 16-18 years). To examine the effects of spinal irradiation on spinal growth independent of growth hormone deficiency we analysed the leg length (LL) minus sitting height (SH) standard deviation score (SDS) and used the cranial group as controls. There was an overall significant difference between the median craniospinal LL-SH SDS (1.98) and the median cranial LL-SH SDS (0.545). Within the craniospinal group there was a significant correlation with age at treatment, but there was no such correlation for the cranial group. After splitting age at treatment into three groups $(0<5,5<10$, and $10-15$ years) there was a significant difference between the LL-SH SDS of the craniospinal and cranial groups for each of the age ranges.

In conclusion, spinal irradiation has a profound effect on spinal growth and the younger the child is when given irradiation the greater the subsequent skeletal disproportion. Our most conservative figures indicate that the eventual loss in height is $9 \mathrm{~cm}$ when irradiation is given at 1 year, $7 \mathrm{~cm}$ when given at 5 years, and $5.5 \mathrm{~cm}$ when given at 10 years.

Impaired growth leading to short stature may complicate the treatment of malignant disease. Radiation induced growth hormone (GH) deficiency and spinal irradiation are two major adverse factors that contribute to the short adult height achieved by many patients treated for brain tumours and acute lymphoblastic leukaemia in childhood. While radiation induced $\mathrm{GH}$ deficiency has been studied quite extensively, ' the effect of irradiation of the spine on spinal growth has rarely been studied. Neuhauser et al suggested that the effects of irradiation on the vertebral bodies were dose and age related. ${ }^{2}$ Probert et al concluded, however, that children receiving spinal irradiation under the age of 6 or at the time of puberty showed particularly pronounced retardation of spinal growth. ${ }^{3}$

In this study of children treated for brain tumours we have analysed the impact of age at spinal irradiation on final height achieved, and estimated the eventual loss in stature attributable to impaired spinal growth.

\section{Patients and methods}

Seventy nine patients (42 males and 37 females) previously treated for a brain tumour in childhood were studied. In all patients a surgical procedure consisting of either partial or complete removal of the primary tumour or biopsy alone was performed. After surgery 37 patients (23 males and 14 females) received craniospinal irradiation, while the remaining 42 (19 males and 23 females) received cranial irradiation alone.

In no child did the primary tumour directly involve the hypothalamic-pituitary axis. The histology of the primary tumour in the craniospinal group was medulloblastoma (26 cases), ependymoma (six), pineal tumour (three), and astrocytoma (two) and in the cranial group was glioma (37), ependymoma (two), angioma (two), and neuroma (one).

In the craniospinal group three radiation schedules had been used for the spinal irradiation. Fifteen children received kilovoltage irradiation with a total dose of $2700 \mathrm{cGy}$ in 17 fractions over 22 days at posterior border or vertebral bodies: the remaining 22 patients received megavoltage irradiation with a total dose of either $3000 \mathrm{cGy}(n=17)$ or $3500 \mathrm{cGy}$ $(n=5)$ in 20 fractions over 27 days to whole vertebra.

The age at which the children in the craniospinal group received irradiation ranged from 1 to 15 years 
and in the cranial group from 2 to 15 years. Subsequently, eight of the children in the craniospinal group and six in the cranial group received treatment with $\mathrm{GH}$ for radiation induced $\mathrm{GH}$ deficiency.

At the time of the study all 79 patients had completed their growth. Their ages ranged from 16 to 30 years. No patient developed sex steroid deficiency that necessitated replacement therapy either as a consequence of radiation induced gonadotrophin deficiency (cranial irradiation) or from direct radiation induced gonadal damage (spinal irradiation). All had undergone puberty spontaneously. Standing and sitting height measurements were performed using a Harpenden Stadiometer and sitting height table. Subischial leg length was obtained by subtracting sitting height from standing height. Standard deviation scores for sitting height and subischial leg length were calculated for chronological age using published tables, ${ }^{+}$with the exception of those aged over 18 years for whom the standard deviation score (SDS) was calculated as if for an 18 year old.

Statistical evaluation was performed using the Mann-Whitney U test, Kruskal-Wallis non-parametric analysis of variance (ANOVA), Spearman's correlation, and linear regression analysis where appropriate.

\section{Results}

The age at irradiation, standing height SDS, sitting height SDS, leg length SDS, and leg length-sitting height (LL-SH) SDS are shown in the Table. There was no significant difference in sex distribution, mean ages, or number of children treated with $\mathrm{GH}$ between the cranial and craniospinal groups.

There was a significant difference between the median standing height SDS of the two groups $(-2.37 v-1 \cdot 14$; Mann-Whitney U test, $\mathrm{p}<0.0(0)(5)$. Within both the craniospinal group (Spearman's $p=0.63, p<0 \cdot 0001)$ and the cranial group (Spearman's $p=0.45, p<0.0(05)$ there was a correlation between standing height SDS and age at irradiation.

There was a significant difference between the median sitting height SDS of the two groups $(-3 \cdot 27$ $v-1 \cdot 19$; Mann-Whitney U test, $\mathrm{p}<0 \cdot 0001)$. Within both the craniospinal group (Spearman's $p=0.62$, $\mathrm{p}<0.001$ ) and the cranial group (Spearman's $p=0.45, p<0.005)$ there was a correlation between sitting height SDS and age at irradiation.

There was no significant difference between the median leg length SDS of the two groups $(-1.12 v$ -0.55 ; Mann-Whitney $U$ test, $p>0 \cdot 1$ ). Within both the craniospinal group (Spearman's $p=0 \cdot 46$, $p<0.005$ ) and the cranial group (Spearman's $p=0.35, p<0 \cdot 05)$ there was a correlation between leg length SDS and age at irradiation.

To concentrate on the impact of spinal irradiation rather than other adverse factors responsible for short stature the LL-SH SDS results were analysed in both groups and by splitting each group into three age divisions $(0<5$ years, $5-10$ years, and $10-15$ years) for cross comparison. There was a significant difference between the median LL-SH SDS of the two groups $(1.98 \vee(0.55$; Mann-Whitney $U$ test, $\mathrm{p}<0.0001)$. These results were reinforced by the significant differences observed for each of the three age groups between the cranial and craniospinal groups. Interestingly, the median LL-SH SDS of the cranial group (().55), as well as the median LL-SH SDS of the craniospinal group (1.98), was significantly different from normal. Within the craniospinal group there was a significant correlation with age at irradiation (Spearman's $p=-0 \cdot 4$, $\mathrm{p}<0.02)$, which was maintained even if the eight children who received treatment with $\mathrm{GH}$ were excluded from analysis; no such correlation existed, however, within the cranial group (Figure).

To see if the three radiation schedules for spinal irradiation had different effects on spinal growth the median LL-SH SDS were compared between the three groups and no significant differences were found.

Finally, a quantitative estimate was made of the loss in eventual stature caused by irradiation of the spine at different ages. Using the linear regression equations shown in the Figure, the LL-SH SDS in both the craniospinal and cranial irradiation groups were calculated at 1,5 , and 10 years of age. In each case, subtracting the LL-SH SDS result for the cranial group from that obtained in the craniospinal group provided the LL-SH SDS difference caused

Table Age at irradiation (months) and standing height, sitting height, leg length, and leg length-sitting height standard deviation scores (SDS) in 79 patients treated with cranial or craniospinal irradiation

\begin{tabular}{|c|c|c|c|c|c|}
\hline \multirow[t]{2}{*}{ Treatment group } & \multirow{2}{*}{$\begin{array}{l}\text { Meam (SI)) age } \\
\text { at irrudiation }\end{array}$} & \multicolumn{4}{|l|}{ Median (range) SDSS } \\
\hline & & Standing height & Sitting he'ight & Legg length & legg length-sitting height \\
\hline
\end{tabular}



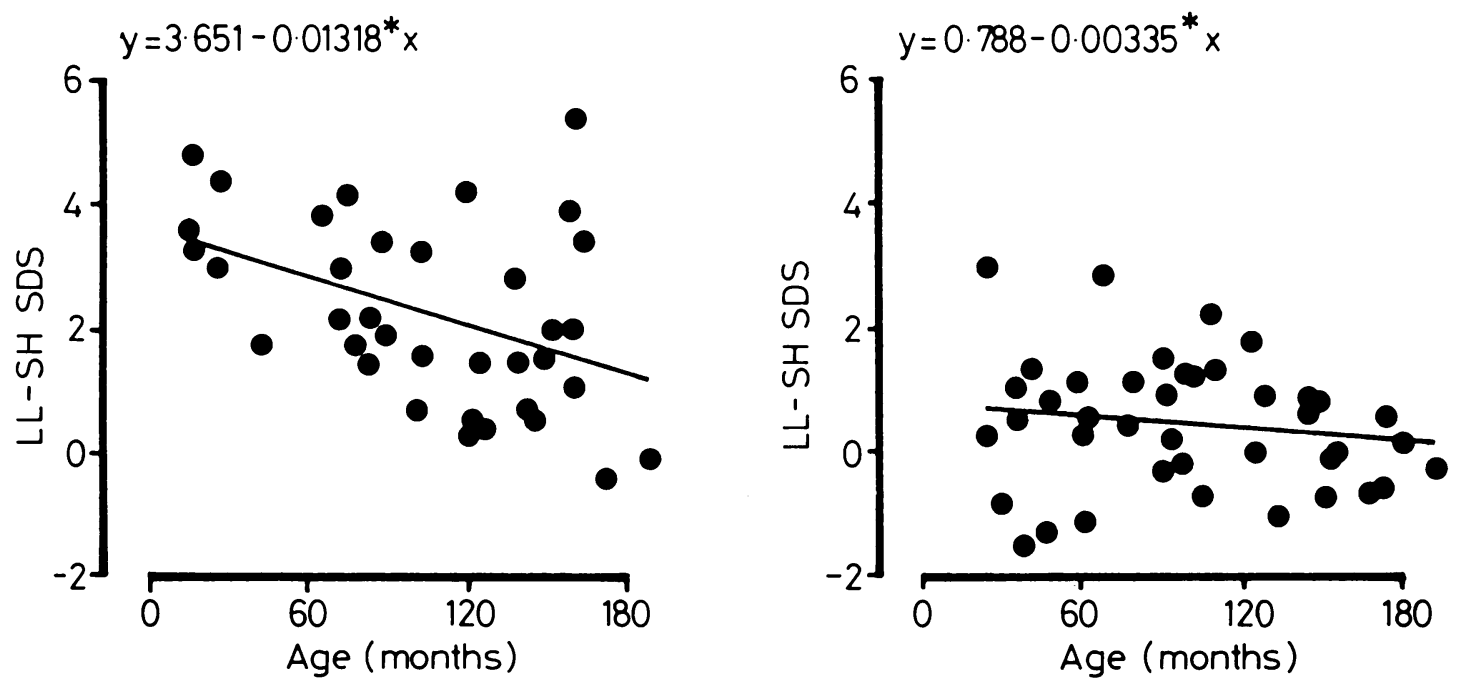

Figure Leglength-sitting height standard deviation score ( $L L-S H S D S)$ versus age at which irradiation was given for the craniospinal (left) and cranial (right) groups. The linear regression equation is stated for both groups $(y=L L-S H S D S$ and $x=$ age in months).

by spinal irradiation. The differences in LL-SH SDS were $2 \cdot 745,2 \cdot 273$, and 1.683 for 1,5 , and 10 years, respectively. If it was assumed that these differences were totally attributable to impaired spinal growth due to spinal irradiation then 'translating' the SDS differences into centimetres of 'lost spinal height' on the standard chart ${ }^{4}$ indicated that the eventual loss in height was $9 \mathrm{~cm}$ if irradiation was given at 1 year, $7 \mathrm{~cm}$ if given at 5 years, and 5.5 centimetres if given at 10 years.

\section{Discussion}

The results of this present study confirm that adults previously treated for a brain tumour were short..$^{5}$ Short stature occurred in those previously treated with cranial irradiation (median standing height SDS $-1 \cdot 14$ ) but was more pronounced in those who received craniospinal irradiation (median standing height SDS $-2 \cdot 37$ ). Two major factors contributing to growth failure were radiation induced $\mathrm{GH}$ deficiency and spinal irradiation. The correlation between either standing height SDS or sitting height SDS and age at irradiation reflects the fact that the younger the age at which GH deficiency or other adverse factors affecting growth occur then the greater the loss in growth potential. Both standing height and sitting height SDS correlations with age at treatment were greater for those children receiving craniospinal rather than cranial irradiation, due to greater impairment in spinal growth seen in younger children.
The conclusion that spinal irradiation was a significant adverse factor in the growth of these children was further supported by the observation that there was no significant difference between the median leg length SDS of the craniospinal and cranial groups.

To disentangle the effects of GH deficiency and spinal irradiation on spinal growth we analysed the LL-SH SDS in both groups. This was necessary for two reasons; firstly, the GH state of many of these patients $(25 \%)$ had not been formally assessed and, secondly, GH deficiency does lead to a short spine but does not cause skeletal disproportion. The LL-SH SDS therefore provided an estimate of the height loss due to spinal irradiation, which could not be calculated with the spinal height SDS data alone.

It was of interest to note that the median LL-SH SDS of the cranial group $(0.545)$ was significantly different from normal $(0)$. This probably reflected the use of the Harpenden Stadiometer for making our measurements, which was not the method used in the construction of the original SDS, which were formulated using an anthropometer ${ }^{4}$ it may have been that this overestimated the sitting height of the children studied. In our calculations, therefore, the cranial group provide a very important body of control data not otherwise available.

Our results in the very young child confirmed the earlier observations of Probert et al that spinal growth was considerably impaired if the spine had been irradiated before 6 years of age ${ }^{3}$ We disagreed with these authors, however, over their conclusion 
that irradiation of the spine during puberty was particularly likely to impair spinal growth. ${ }^{3}$ The younger the child at irradiation the greater the loss in growth potential, from $9 \mathrm{~cm}$ at 1 year to $5.5 \mathrm{~cm}$ at 10 years.

Medulloblastoma and ependymoma exfoliate cells into the cerebrospinal fluid, and seeding may occur most commonly in the spinal canal. Spinal irradiation is given to prevent spinal relapse of disease. It can be seen, however, that the price for such protection is high in terms of loss of growth potential. It may be that the same 'spinal protection' can be afforded by a lower dose of spinal irradiation. Such studies need to be undertaken in the future.

We thank the Cancer Research Campaign for finaricial support.

\section{References}

1 Shalet SM. Irradiation induced growth failure. Clin Endocrinol Metab 1986;15:591-606.

2 Neuhauser EBD, Wittenborg MH, Bereman CZ, Cohen J. Irradiation effects of roentgen therapy on the growing spine. Radiology 1952;59:637-50.

${ }^{3}$ Probert JC, Parker BR, Kaplan HS. Growth retardation in children after megavoltage irradiation of the spine. Cancer 1973;32:634-9.

4 Tanner JM, Whitehouse RH. Standards for sitting height and subischial leg length from birth to maturity: British children 1978. Hertford: Castlemead Publications, 1979.

${ }^{5}$ Bamford FN, Morris Jones PH, Pearson D, Ribeiro GG, Shalet SM. Beardwell CG. Residual disabilities in children treated for intracranial space occupying lesions. Cancer 1976;37: 1149-51.

Correspondence to Dr S M Shalet, Department of Medicine and Endocrinology, Christie Hospital and Holt Radium Institute, Wilmslow Road, Withington, Manchester M20 9BX. England.

Received 12 December 1986 